\title{
Reversal Reactions Observed in Two Cases of Subpolar Lepromatous Leprosy
}

\author{
Namisato $\mathrm{M}^{1 *}$ and Ogawa $\mathrm{H}^{2}$ \\ ${ }^{1}$ Department of Dermatology, Auen Poly-Clinic, Japan \\ ${ }^{2}$ Chief Director of Juntendo University, Japan
}

*Corresponding author: Masako Namisato, Director of Auen Poly-Clinic, 1037-1 Nakatomi Tokorozawa-city, Saitama-prefecture, 359-0002 Japan, Tel: +81-4-2990-5818; Fax: +81-42990-5828; Email: namima@auen.jp

\section{Case Report}

Volume 5 Issue 1

Received Date: January 06, 2020

Published Date: January 28, 2020

DOI: $10.23880 /$ cdoaj-16000200

\section{Abstract}

We must always be cautious about leprosy reactions that can cause severe, irreversible deformities when treating leprosy. We studied the clinical course of two patients with subpolar lepromatous leprosy. The first patient was newly diagnosed and the second had relapsed. The latter had drug resistance-related mutations to three drugs, diaminodiphenyl sulfone, rifampicin, and ofloxacin.

Patient 1 had a heavy bacterial load and type 2 leprosy reaction (erythema nodosum leprosum) before starting chemotherapy. After 2 weeks of starting the chemotherapy, he developed a severe reversal reaction (RR), which sustained for approximately 6 months.

The bacterial load of patient 2 was not as heavy as that of patient 1 . He developed RR about 1.5 years after starting the chemotherapy and it was sustained for about 1 year. The severity of RR was lower than in patient 1 .

About 3 years after beginning the chemotherapy, when their bacterial loads had greatly decreased, both patients developed RR again, with nerve trunk injury. Thereafter, their leprosy was completely cured without any residual motor dysfunction. The appropriate length of the follow-up period should be further investigated.

Keywords: Leprosy reactions, Reversal reaction, Chemotherapy, Bacterial load, Duration of chemotherapy

Abbreviations: RR: Reversal Reaction; LLs: Subpolar Lepromatous Leprosy; BI: Bacterial Index; AFB: Acid-Fast Bacilli; MINO: Minocycline; DDS: Diaminodiphenyl Sulfone; RFP: Rifampicin; OFLX: Ofloxacin; CAM: Clarithromycin; CLF: Clofazimine; LVFX: Levofloxacin.

\section{Introduction}

Most of the leprosy-related disabilities are caused by leprosy reactions. Early diagnosis, timely initiation of antiinflammatory measures, and effective chemotherapy are essential. There are 2 types of reactions, type 1 leprosy reaction or reversal reaction (RR) and type 2 leprosy reaction or erythema nodosum leprosum (ENL).

The RR represents an episode of acute cell-mediated hypersensitivity response to some antigens related to Mycobacterium leprae (M. leprae). This may occur in the borderline cases of leprosy in which the natural course of the disease is followed without chemotherapy. However, in most cases of subpolar lepromatous leprosy (LLs), RR usually develops following the chemotherapy. Depending on the 
Clinical Dermatology Open Access Journal

decrease of the host's bacterial load caused by chemotherapy, the immunological status can become more active against $M$. leprae and RR can develop at this time [1].

ENL is considered as a classical example of an immune complex disease known to affect patients with borderline leprosy and LL. However, based on our experience, it affects only LL, especially after WHO/MDT [2] was introduced as the standard regimen for leprosy.

We studied the RRs observed in the two patients with LLs, whose bacteriological status was different. The time of development, the severity, and the duration of each RR were compared between the two.

The bacterial load is shown by the bacterial index (BI) of a skin smear [3] and/or $M$. leprae-specific anti-PGL-I antibody titer $[4,5]$.

\section{Case Report}

\section{Patient 1}

Patient 1 was a 35-year-old Japanese man, born and raised in Paraguay.

He visited a local hospital when having generalized erythema, some intradermal nodules, high fever, and severe malaise. As reported by him, some skin lesions developed 1 year ago. Pathological findings of erythematous lesions on his forehead revealed clustered foamy macrophages containing abundant acid-fast bacilli (AFB) and he was diagnosed with LL.

He also had some pea-sized asymptomatic umbilicated scar-forming lesions on his extremities (Figure 1). They were reported as "Transepidermal elimination of lepromatous granuloma: a mechanism for mass transport of viable bacilli" [6].
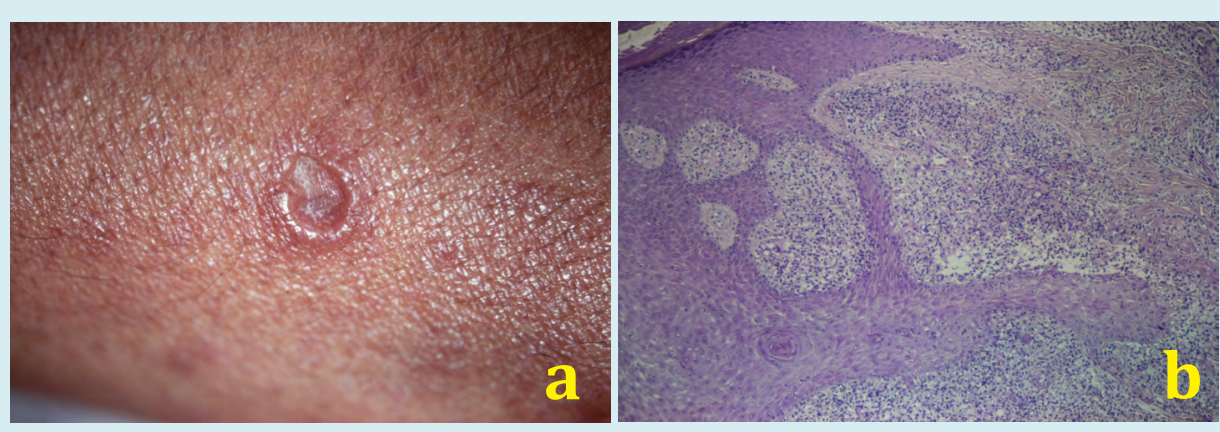

Figure 1: a: Pea-sized umbilicated lesion. b: Transepidermal elimination of lepromatous granuloma (HE $\times 100)$.

After diagnosis, minocycline (MINO) $200 \mathrm{mg} /$ day for 1 week was administered and he was then referred to our institute. After 2 weeks of initiating MINO, his extremities were swollen on the first examination. Bilateral ulnar nerves were thick and tender. Moderate uveitis was also found. The maximum BI was 5+. Pathological findings of a nodule on his buttock revealed a lepromatous granuloma composed of foamy macrophages having globi of AFB, mostly rod shaped, and some were degenerated. Vacuolated spaces were stuffed with massive AFB. A laminated perineurium was also found (Figure 2).

WHO/MDT for multibacillary leprosy [7] was then started. Three days after starting MDT, severely inflamed skin lesions were observed all over his body and severe neuritis developed on the extremities, accompanied with high fever (Figure 3). Thalidomide was not effective but prednisolone $30 \mathrm{mg} /$ day (PSL) was very effective. Upon nerve biopsy, severely infiltrated lymphocytes, neutrophils, epithelioid cells, and multinucleated giant cells were found and RR was confirmed. Large vacuoles stuffed with abundant AFB were also noticed (Figure 4). The RR continued for about 6 months.

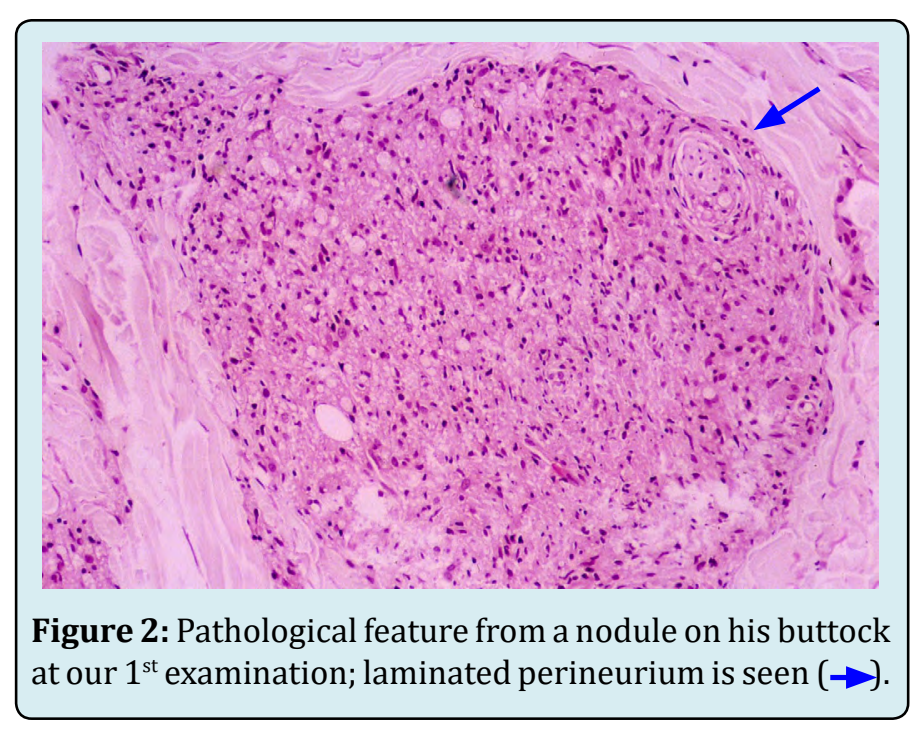



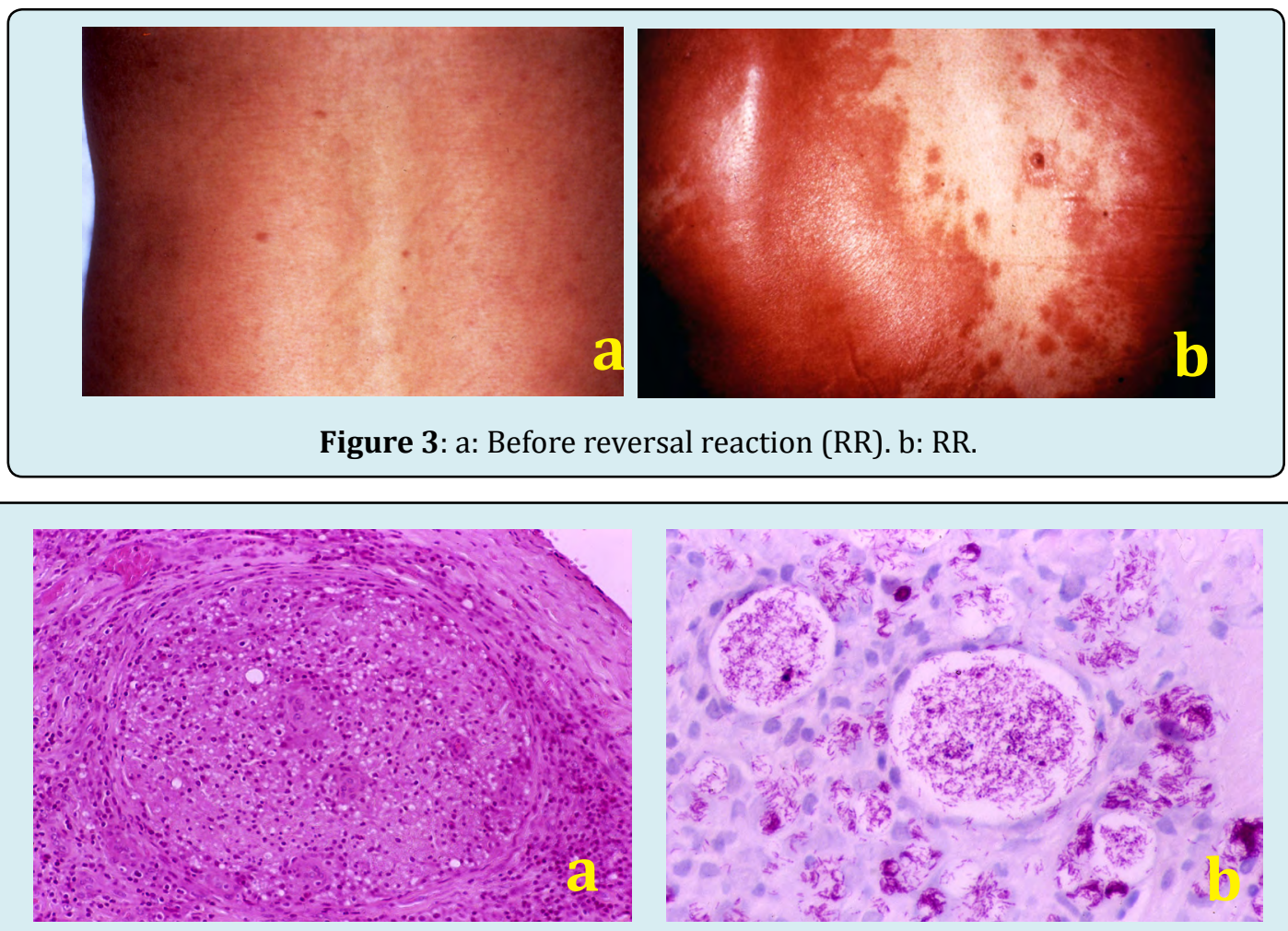

Figure 4: a: Nerve biopsy (HE $\times 100)$. Severely infiltrated lymphocytes, neutrophils, epithelioid cells as well as thickened perineurium are seen.

b: Fite stain $\times 400$. Vacuolated spaces are stuffed with massive degenerated AFB.

After 2 years and 2 months of MDT, the maximum BI was $3+$. Because of the insufficient decrease of bacterial load, the second regimen composed of $100 \mathrm{mg} /$ day diaminodiphenyl sulfone (DDS) and $100 \mathrm{mg} /$ day MINO was started. After 10 months of starting the second regimen, he developed RR again, with right facial nerve palsy. Prompt administration of $40 \mathrm{mg} /$ day PSL was started, which was then tapered. The patient completely recovered in 1.5 months (Figure 5).

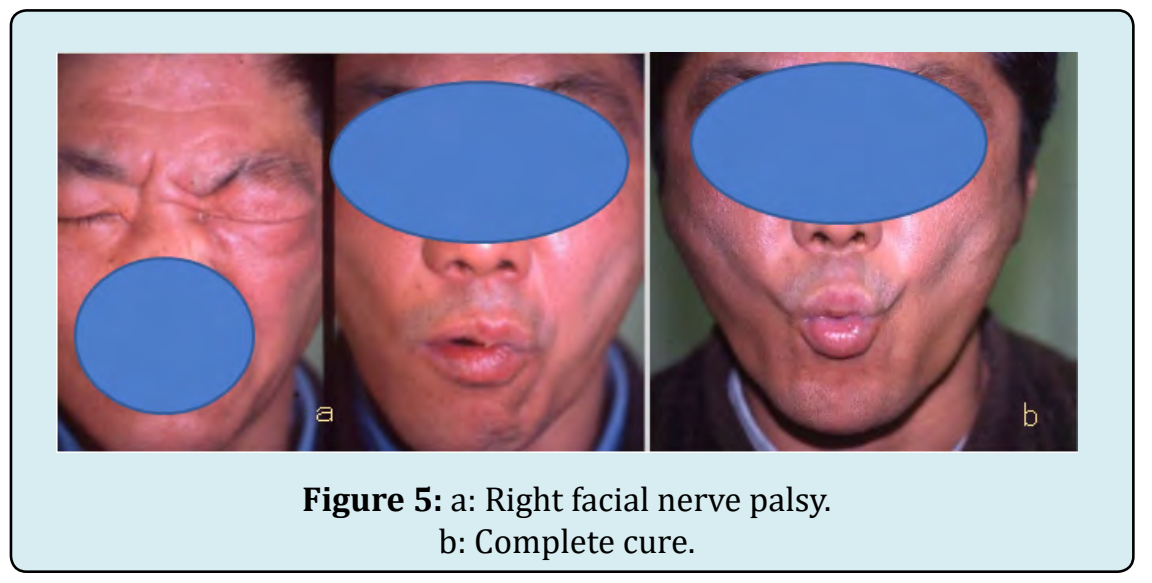

\section{Patient 2}

The second patient was a 68-year-old Japanese man. He was diagnosed with leprosy when he was 16 years of age. He had been treated with various sulfones until he turned 27 years old. After 6 years, his disease relapsed; DDS and rifampicin (RFP) were prescribed and ofloxacin (OFLX) was added later. He entered remission approximately at the age of 43 years.

On the first examination at our clinic, his face was red 


\section{Clinical Dermatology Open Access Journal}

and edematous. Numerous red macules of varying sizes were disseminated, which were confluent on his trunk and extremities. There were no apparent lepromatous nodules. He discovered some lesions, which gradually increased, 10 months earlier.
The skin smear of red macules was 2+ using the fluorescent staining method, equivalent to Gaffky 5. The pathological findings showed lepromatous granuloma with proliferated foamy macrophages containing many AFB and LL diagnosis was confirmed (Figure 6).
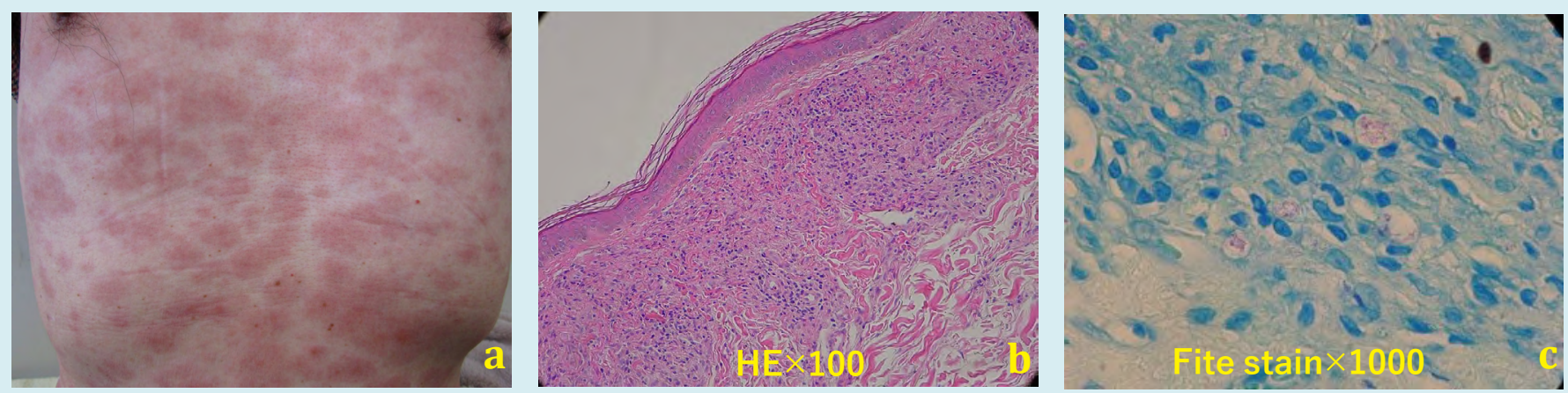

Figure 6: a: Clinical feature on the 1st examination of Patient 2.

b, c: Lepromatous granuloma and foamy macrophage containing rod shaped acid-fast bacilli

In contrast, drug resistance assays were performed and surprisingly, drug resistance-related-mutations to three drugs, DDS, RFP, and OFLX, were found.

Therefore, a combination of MINO, clarithromycin (CAM), and clofazimine (CLF) was started. CLF was administered for only 15 days to avoid severe skin coloration. For the first 4 months, levofloxacin (LVFX) was also included for possible intermixed new quinolone-sensitive bacilli. MINO and CAM were given daily for about 1 year and then tapered to twice or once a week. After 2 weeks of starting the treatment, the edematous redness on his face disappeared, and 5 months later most skin lesions became flattened. After about 1.5 years, suddenly increased redness of previously existing lesions was noticed, and similar symptoms continued for about 1 year. Each episode was well controlled with nonsteroidal anti-inflammatory drugs and a small amount of PSL, 10 20 mg/day, for less than 2 weeks.

Severe neuralgia of the median nerve occurred 2 years and 10 months later. Prompt administration of $30 \mathrm{mg} /$ day PSL was started and was then tapered. After 6 weeks, RR disappeared without any resulting sequelae.

About 5 years later, the skin smear was negative, and the treatment was discontinued. ENL was not observed during the entire clinical course. Details of this case are reported in the referenced paper [8].

The clinical courses of the two patients are shown in Tables $1 \& 2$.

Table: 1

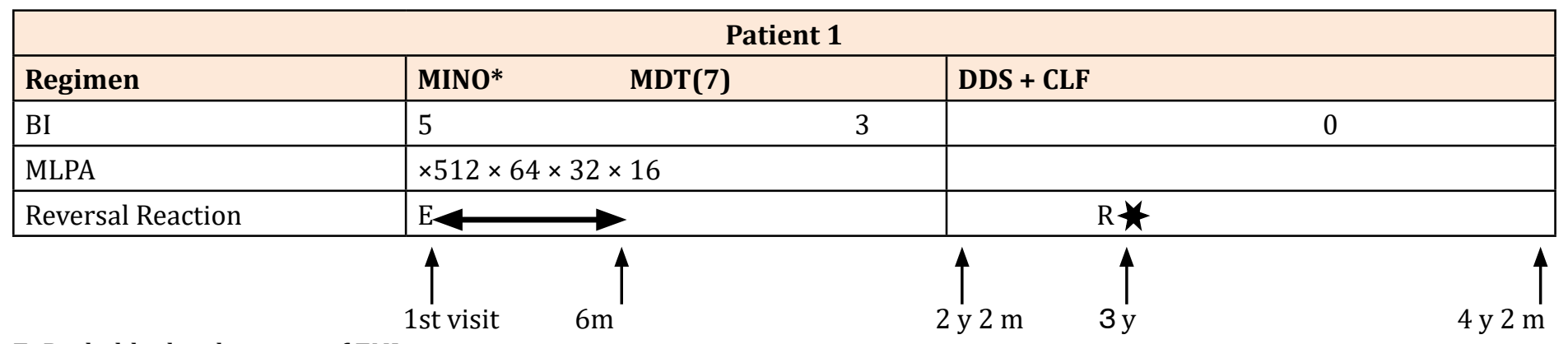

E: Probable development of ENL.

BI: Maximum Bacterial Index [3].

MLPA: Mycobacterium leprae particle agglutination test [5].

MNO*: MINO was given for 1 week. 


\section{Clinical Dermatology Open Access Journal}

Table: 2

\begin{tabular}{|c|c|c|c|c|c|}
\hline \multicolumn{6}{|c|}{ Patient 2} \\
\hline \multirow[t]{2}{*}{ Regimen } & \multicolumn{2}{|l|}{ NQ } & \multicolumn{2}{|c|}{ MINO $\cdot$ CAM $\cdot$ CLF } & \\
\hline & daily & 2/wee & & 1/week & \\
\hline ELISA & 0.367 & 0.336 & 0.128 & & 0.054 \\
\hline Reversal Reaction & & & & $\mathrm{R} *$ & \\
\hline
\end{tabular}

ELISA: Enzyme linked immunosorbent assay, the titer of M. leprae-specific anti-PGL-I antibody (4). C Cutoff: 0.15.

Tables 1 \& 2: Clinical course of two patients

Each regimen, time and duration of reversal reaction (RR), and change of bacterial load (BI, ELISA, MLPA) are shown.

y, m: Year, month

NQ: New quinolone, used for 4 months

MINO: Minocycline

DDS: Diaminodiphenyl sulfone

CLF: Clofazimine, used for only 15 days

CAM: Clarithromycin

R: Reversal Reaction

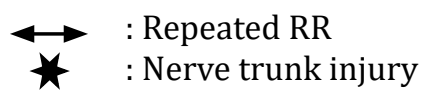

\section{Discussion}

Patient 1 had full-fledged LL. The symptoms of high fever and severe malaise before starting the chemotherapy were suspected to be symptomatic of ENL. The pathological findings from our first examination showed a laminated perineurium, which is characteristic in the borderline group of leprosy and LLs. Therefore, he was diagnosed with LLs and we were cautious of possible RR. Severe RR developed only 3 days after starting MDT and continued for about 6 months and his condition was quiescent thereafter.

Patient 2 had a lower bacterial load than patient 1 and his RR developed about 1.5 years after starting the chemotherapy. It continued for 1 year but the severity was much less than in patient 1.

In both the cases, the patients developed RR again with severe neural symptoms about 3 years after starting the chemotherapy. Based on the change of the BI or anti-PGL-I antibody titer, their bacterial load had greatly decreased before developing their final RR. In these two cases, interestingly, the final RR accompanied severe nerve trunk injury.

In the leprosy treatment, the necessary duration of chemotherapy and follow-up period are important aspects for discussion. From our experience, the final RR can develop well after a decreased bacterial load is seen, followed by complete cure. RR can therefore be a "rite of passage."

\section{References}

1. Yawalkar SJ (1994) Reactions in Leprosy. In: Yawalkar SJ (Ed.), Leprosy. $6^{\text {th }}$ (Edn.), CIBA-GEIGY, Limited, Basle, Switzerland, pp: 83-91.

2. World Health Organization Study Group (1982) Chemotherapy of leprosy for control programmes. World Health Organization Technical Report Series No.675, Geneva.

3. Ridley DS, Hilson GRF (1967) A logarithmic index of bacilli in biopsies I. Method. Int J of Lepr 35(2): 184-186.

4. Hunter SW, Fujiwara T, Brennan PJ (1982) Structure and antigenicity of the major specific glycolipid antigen of Mycobacterium leprae. J Biol Chem 257(24): 1507215078.

5. Izumi S, Fujiwara T, Ikeda M, Nishimura Y, Sugiyama K, et al. (1990) Novel gelatin particle agglucination test for serodiagnosis of leprosy in the field. J Clin Microbiol 28(3): 525-529.

6. Namisato M, Kakuta M, Kawatsu K, Obara A, Izumi S, et al. (1997) Transepidermal elimination of lepromatous 
granuloma: a mechanism for mass transport of viable bacilli. Lepr Rev 68(2): 167-172.

7. World Health Organization Study Group (1994) Chemotherapy of leprosy. WHO Technical report series 847, Geneva.
8. Namisato M, Ogawa H (2019) Essential aspect of leprosy classification and clinical course of 5 leprosy patients: including four relapsed cases. Clin Dermatol J 4(4): 000187. 